\title{
Algebraic approach to quantum black holes: logarithmic corrections to black hole entropy
}

\author{
Gilad Gourt \\ Racah Institute of Physics, Hebrew University of Jerusalem, \\ Givat Ram, Jerusalem 91904, ISRAEL \\ and \\ Theoretical Physics Institute, Department of Physics, University of Alberta, \\ Edmonton, Canada T6G 2J1
}

\begin{abstract}
The algebraic approach to black hole quantization requires the horizon area eigenvalues to be equally spaced. As shown previously, for a neutral nonrotating black hole, such eigenvalues must be $2^{n}$-fold degenerate if one constructs the black hole stationary states by means of a pair of creation operators subject to a specific algebra. We show that the algebra of these two building blocks exhibits $U(2) \equiv U(1) \times S U(2)$ symmetry, where the area operator generates the $U(1)$ symmetry. The three generators of the $S U(2)$ symmetry represent a global quantum number (hyperspin) of the black hole, and we show that this hyperspin must be zero. As a result, the degeneracy of the $n$-th area eigenvalue is reduced to $2^{n} / n^{3 / 2}$ for large $n$, and therefore, the logarithmic correction term $-3 / 2 \log A$ should be added to the Bekenstein-Hawking entropy. We also provide a heuristic approach explaining this result, and an evidence for the existence of two building blocks.
\end{abstract}

*E-mail: gour@cc.huji.ac.il, gilgour@Phys.UAlberta.CA 


\section{INTRODUCTION}

The flurry of activity on the theory of black holes in general relativity illuminated a very deep and fundamental relationship between gravitation, thermodynamics and quantum theory [1]. The bedrock of this connection is the notion of back hole entropy [2]. Bekenstein's identification of the horizon area with black hole entropy has provided a criterion for the success of any candidate quantum theory of gravity. Today we are presented with several generic quantum gravity theories (superstring and D-brane theory [3], loop quantum gravity [4], canonical quantum gravity [5,6,7]) all of which reproduce the black hole entropy from a microscopic counting of states. Despite this success, there is as yet no generally accepted theory of quantum gravity; other conditions are needed to provide further tests on theories which purport to represent quantum gravity.

In [8] it is shown that a sufficient condition for establishing the identification $S_{B H} \propto A$ is that the complete system, black hole-Hawking radiation, can be characterized by classical thermodynamics. Furthermore, it is shown (assuming the first law of black holes mechanics) that any nonlinear correction to the black hole entropy must be quantum mechanical in nature. For example, in a Reissner-Nordstrom black hole, the dimensionless function $\Phi_{T} \equiv$ $(\partial M / \partial Q)_{T}$ (which can be interpreted as the electric potential on the horizon when the black hole is in equilibrium with a surrounding heat bath) can be expanded in powers of $\hbar$ where the leading term $O\left(\hbar^{0}\right)$ is taken to be classical. It is shown in 8 that higher order terms in $\hbar$ appear if and only if $S^{\prime \prime}(A) \neq 0$, where $S(A)$ is the entropy of the black hole and $A$ is the horizon surface area. Therefore, the leading order corrections to the Bekenstein-Hawking entropy (if they exist) will provide a further insight in the description of quantum effects in black holes.

Very recently, several authors made an attempt in this direction. For example, Kaul and Majumdar [9] (see also [11, 12]) have calculated the lowest order corrections to the Bekenstein Hawking entropy in a particular formulation of quantum loop gravity [10]. In this approach, the black hole horizon is treated as a boundary spacetime which is quantized within the "quantum geometry" program. They have found a $-3 / 2 \log A$ correction term to the black hole entropy. Carlip [13] has found exactly the same correction term for the BTZ black hole by using the corrected version of the asymptotic Cardy formula. He has also shown that similar logarithmic corrections appear in all black holes whose microscopic degrees of freedom are described by an underlying conformal field theory. Recently, Das, Majumdar and Bhaduri [14] have computed the leading order corrections to the entropy of any thermodynamic system due to small statistical fluctuations around equilibrium. These corrections also turn out to be logarithmic in the horizon area when applied to black holes, and equal to $-3 / 2 \log A$ when applied to the BTZ black hole. More recently, Gupta et al. [15] obtain the same correction term by using a scalar field as a simple probe of the background geometry of a massive Schwarzschild black hole. Here, we shall obtain exactly the same logarithmic correction by using a simple heuristic scheme, and also by exploiting the algebraic approach [16,17]. We further argue that positive logarithmic corrections might also be required in the Bekenstein-Hawking entropy if the horizon area fluctuations are included. This may shed some light on why other authors [18, 19, 20, 21, 22, 23, 24, 25, 26, 27] have obtained logarithmic corrections with different coefficients.

Among the simplest questions which can be asked, and which can also provide condi- 
tions on a quantum theory of gravity, is what is the nature of the energy spectrum of a black hole. The remarkable observation that the horizon area behaves as a classical adiabatic invariant [28,29, 30 provided the first clue in this direction. In the spirit of Ehrenfest principle, Bekenstein [28,31] conjectured that the horizon area of a non extermal quantum black hole has a discrete eigenvalue spectrum. He also conjectured that the spacing between area eigenvalues is uniform [2,31] since the assimilation of a quantum particle into a KerrNewman black hole carries a minimal "cost" $\sim \hbar$ of area increase, which does not depend on the black hole parameters. The discrete nature of the eigenvalue spectrum for the horizon area is also supported by the loop quantum gravity [4], but this last theory suggests a rather complicated eigenvalue spacing. If the area spectrum is equally spaced, the classical relation $A=16 \pi M^{2}(c=G=1)$ for a Schwarzschild black hole implies the mass spectrum $M \sim \sqrt{\hbar n}$ for it, where $n=1,2, \cdots$. This type of spectrum has subsequently been obtained by many authors (see the list in [17] and Ref. [32]).

The heuristic approach mentioned above is far from giving a complete description of quantum effects in black holes. In order to illuminate the inner structure of a black hole, an algebraic approach to black hole quantization has been developed [16,17]. Analogously to quantum loop gravity [4, in this approach one seeks to determine the spectra of relevant observables in the theory by their algebra. However, in the algebraic approach it is assumed that each separate black hole state, which one assumes comes from a discrete set, is created from a "black hole vacuum" $|v a c\rangle$ by a certain "creation" operator:

$$
|n, s\rangle=\hat{R}_{n s}|v a c\rangle \text {. }
$$

Here $|n, s\rangle$ is a one (non-rotating and neutral) black hole state with area $a_{n} ; s=0, \ldots, g_{n}-1$ distinguishes between different states with the same area, where $g_{n}$ is the degeneracy of the said states. It was shown in 16] that the algebra of the various $\hat{R}$ operators together with the horizon area observable (in [16] the angular momentum and charge observables are also included) implies that the spectrum of $\hat{A}$ is equally spaced:

$$
a_{n}=a_{0} n ; \quad n=1,2,3, \cdots
$$

where $a_{0}$ is a positive constant proportional to $\hbar$.

More recently [17], the $\hat{R}$ operators where constructed from a small number of more fundamental "building blocks" out of which the whole algebra follows. It was assumed for simplicity that the first area level has two independent quantum states, say $|1,0\rangle$ and $|1,1\rangle$, so that the corresponding two building blocks for these states are given by:

$$
\hat{a} \equiv \hat{R}_{11} \quad \text { and } \quad \hat{b} \equiv \hat{R}_{12}
$$

It was shown that if one builds the $\hat{R}$ operators as products involving only these two noncommuting operators, the degeneracy of the area levels is given by the exponential law $g_{n}=2^{n}$. This was the first time that a formal proof has been given for this law.

The exponential law $g_{n}=k^{n}(k=2,3, \ldots)$ was first proposed by Mukhanov [33] (see also Ref. [34]) where it was assumed that the entropy of the black hole corresponds to the degeneracy of the area levels Eq. (2). Since then quite a few heuristic ways of understanding the exponential growth of degeneracy have been proposed [31, 34, 35, 36, 37, 38]. One of the simplest views to get the quantization law Eq. (2) suggests that the horizon may be regarded 
as parceled into $n$ patches of area $a_{0}$. If each can exists in $k$ different quantum states, then the law $g=k^{n}$ is immediate [31]. However, as we shall see here, if one includes the hyperspin or the global quantum number of these patches, the exponential law (for $k=2$ ) is replaced by

$$
g_{n}=\left(\begin{array}{c}
n \\
n / 2
\end{array}\right)-\left(\begin{array}{c}
n \\
n / 2+1
\end{array}\right) \approx \frac{2^{n}}{n^{3 / 2}}
$$

for $n \gg 1$. We shall obtain this result also in a more formal way using the algebraic approach where the hyperspin appears as the generator of the symmetry between the two building blocks in Eq. (3). Furthermore, in the Appendix, we start with $k=3$ (equivalent to three building blocks) and find in this case that $g_{n} \approx \frac{3^{n}}{n^{2}}$ which corresponds to a $-2 \log n$ correction term. We shall mention here, that by using a holographic point of view, Das, Kaul and Majumdar [11] have obtained the same result. However, in their formalism the left hand side of Eq. (4) is replaced by the number of $S U(2)$ singlet states contributing to the entropy of an isolated horizon (which is described by the boundary degrees of freedom of a three dimensional Chern Simons theory), and $n$ is replaced by the number of vertices of the network.

The physical interpretation of the building blocks $\hat{a}$ and $\hat{b}$ may be elucidated by comparison with other approaches. It was shown [39] that the algebraic approach and the approach involving quantization on a reduced phase space [40] are similar. However, it is still not clear how the degeneracy appears in the later approach. Here, we shall argue that one of the building blocks is associated with the boundary at infinity through the ADM mass $M$ and its conjugate $P_{M}$ (the time separation at special infinity), and the other with the boundary at the horizon via $A$ and its conjugate $\Theta$ (the "opening angle", see Ref. ( 41])). This also gives an argument for setting $k=2$.

The paper is organized as follows: In Sec. III, we develop a heuristic approach to calculate the $-3 / 2$ logarithmic correction term. In Sec. III, we provide a physical interpretation to the building blocks, by a comparison with the reduced phase space approach. In Sec. IV, the hyperspin is defined within the algebraic approach, and the degeneracy of the area levels in the hyperspin representation is obtained. We conclude with a discussion (Sec. V). The Appendix generalizes the results to the case of three building blocks.

\section{HEURISTIC SCHEME AND LOGARITHMIC CORRECTIONS}

Since any correction to the black hole entropy formula, $S_{\mathrm{bh}}=\eta A$, must be quantum mechanical in nature [8], the leading order corrections to the black hole entropy (if they exist) will provide a further insight into the interplay between general relativity and quantum mechanics. Furthermore, as one reduces the size of the black hole, these corrections may play an important role and even change completely the semi-classical description.

In previous work [26], we have found a positive logarithmic correction $1 / 2 \log A$ to the Bekenstein-Hawking entropy by considering the Schwarzschild black hole as a grand canonical ensemble, with the Hamiltonian (the ADM mass) and the horizon surface area, separately, as observable parameters. Also Kastrup [24], Mäkelä and Repo [25] and Obregon, Sabido and Tkach [27] obtained exactly the same positive correction. More recently, Major 
and Setter [23] obtained the correction term $\log A$ using a model which is motivated by the formulation of geometry in loop quantum gravity (they also describe the black hole in terms of a grand canonical ensemble). All these approaches predict positive corrections due to the area fluctuations. The area fluctuations increase the uncertainty, and therefore, the number of microstates which describe the black hole. However, the logarithmic corrections which correspond to corrections in the degeneracy $g(n)=k^{n}$ are not positive. We shall give now a simple heuristic argument, based on the equally spaced area spectrum, which yields the correction term $-3 / 2 \log A$ (in section 4 we shall derive this result from the algebraic approach). This term was obtained by several authors 110,11,12, 13, 14, 15 using a variety of techniques.

In several models of black holes, it is assumed that the black hole consists of elementary components contributing additively to its area. In quantum loop gravity, these components are described by the Wilson lines of the Ashtekar's connection $A_{\mu}^{a}$ (see for example Ref. [42]). In $M$-theory [43, the components are described by the $D_{0}$-branes. Here, we consider the equally spaced area spectrum, where the horizon may be regarded as parceled into $n$ patches (components) of area $a_{0}$. In this heuristic view, these patches are localized. Thus, it is assumed that each cell has the same number of quantum states due to rotational symmetry. For simplicity, we assume that each cell can exist in 2 different quantum states.

Since the elementary components (the patches) can exist in two states, in quantum mechanics they can be described by $1 / 2$-spin entities. These are here called "hyperspins" to distinguish them from spin; i.e. the 1/2-hyperspin which is associated with each patch describes its two different quantum states. The 1/2-hyperspin observable associated with the $i$ 'th patch is denoted by $\overrightarrow{\mathbf{h}}^{(i)} \equiv\left(\hat{h}_{1}^{(i)}, \hat{h}_{2}^{(i)}, \hat{h}_{3}^{(i)}\right)$ where the total hyperspin of a black hole with area $A=a_{0} n$ is given by

$$
\overrightarrow{\mathcal{J}}=\sum_{i=1}^{n} \overrightarrow{\mathbf{h}}^{(i)}
$$

Since $\overrightarrow{\mathcal{J}}$ represents a macroscopic feature of the black hole, it should have played an important role also in the classical description. However, according to Wheeler's no-hair principle, the Schwarzschild black hole is parametrized only by its mass. This leaves us no choice but to set the total hyperspin of the Schwarzschild black hole to zero. Furthermore, by analogy with a system of 1/2-spin particles, it seems reasonable that the energy (mass) should depend on $\mathcal{J}$. If so, the relation $A=16 \pi M^{2}$ holds true only in the classical limit where $\mathcal{J}=0$. This motivates the idea that the Schwarzschild black hole should be considered as a grand canonical ensemble [23,26].

The number of microstates with area $A=a_{0} n$ is $g(n)=2^{n}$. This follows from the two quantum states each cell can exist in. However, the number of microstates $g(n, \mathcal{J}=0)$ with area $A=a_{0} n$ and zero hyperspin $\mathcal{J}=0$ is smaller. A simple calculation (see section [V] shows that

$$
g(n, \mathcal{J}=0)=\frac{1}{1+1 / 2 n}\left(\begin{array}{c}
n \\
1 / 2 n
\end{array}\right) .
$$

Note that $n$ must be even since $\mathcal{J}=0$. Using Stirling's formula, we find in the classical limit $n \rightarrow \infty$ that 


$$
g(n, \mathcal{J}=0) \approx 2^{n} / n^{3 / 2} .
$$

This yields a correction term $-3 / 2 \log n$ to the Bekenstein-Hawking entropy. Moreover, Eq. (6) shows clearly that as one reduces the size of the black hole, the semi-classical description is completely changed. For example, an elementary black hole with area $A=2 a_{0}$ has zero entropy.

In conclusion, logarithmic corrections to the black hole entropy may be classified into two types: positive corrections, which appear due to the area fluctuations, and negative corrections, which appear as a result of corrections to the number of microstates that describe a black hole with a definite horizon area.

\section{A PHYSICAL INTERPRETATION OF THE BUILDING BLOCKS}

In previous work [17], it was suggested that the algebra of black holes observables, can be constructed from two basic operators (building blocks) $\hat{a}$ and $\hat{b}$, satisfying the following commutation relation:

$$
\begin{aligned}
& {[\hat{A}, \hat{a}]=a_{0} \hat{a}, \quad[\hat{A}, \hat{b}]=a_{0} \hat{b}} \\
& {\left[\hat{a}^{\dagger}, \hat{a}\right]=\left[\hat{b}^{\dagger}, \hat{b}\right]=1+\alpha \hat{A} \equiv 1+w \hat{N}} \\
& {\left[\hat{a}^{\dagger}, \hat{b}\right]=\left[\hat{b}^{\dagger}, \hat{a}\right]=0}
\end{aligned}
$$

where $\hat{N} \equiv \hat{A} / a_{0}$ is a dimenssionless number operator. The commutation relation between $\hat{a}$ and $\hat{b}$ may be complicated but it does not matter for our analysis because the degeneracy is determined by relations (8-10) only. It was shown that this algebra leads to exactly $2^{n}$ independent states with the same area eigenvalue $a_{0} n$. This degeneracy corresponds to the entropy of the black hole; in the next section the logarithmic correction term $-3 / 2 \ln n$ will be obtained.

The physical interpretation of the building blocks $\hat{a}$ and $\hat{b}$ may be found by comparison with other approaches. For example, in [39] it was shown that the algebraic approach and the approach involving quantization on a reduced phase space of collective coordinates of a black hole are similar. In the reduced phase space approach [40], one starts, still classically, with an effective action of the form [44]

$$
I=\int d t\left(P_{M} \dot{M}-H(M)\right)
$$

where $M$ is the mass and $P_{M}$ its conjugate momentum (here we consider non-rotating, neutral black holes). The conjugate momentum $P_{M}$ has the interpretation of the difference between the Schwarzschild times at left and right infinities 445] and, according to Euclidean quantum gravity [46], it is periodic with a period which is inverse the Hawking temperature $T_{H}$. The canonical transformation

$$
\begin{aligned}
X & =2 M \cos \left(2 \pi P_{M} T_{H} / \hbar\right) \\
\Pi_{X} & =2 M \sin \left(2 \pi P_{M} T_{H} / \hbar\right)
\end{aligned}
$$

implies that the horizon area $A=16 \pi M^{2}$ can be written in the form $A=4 \pi\left(X^{2}+\Pi_{X}^{2}\right)$. Thus, after quantization $\left(X \rightarrow \hat{X}, \Pi_{X} \rightarrow \hat{\Pi}_{X}\right.$ where $\left.\left[\hat{X}, \hat{\Pi}_{X}\right]=i \hbar\right)$ the area observable 
exhibits an equally spaced area spectrum. Another way to obtain this result, is to perform the canonical transformation

$$
\left(M, P_{M}\right) \rightarrow(A / 8 \pi, \Theta)
$$

where $\Theta \equiv \kappa P_{M}$ and $\kappa$ is the surface gravity. Note that $\Theta$ has periodicity of $2 \pi$ and that it is the canonical conjugate of $A / 8 \pi$. Thus, after quantization $A / 8 \pi$ is represented by a number operator.

One can identify one of the building blocks, say $\hat{a}$, with the creation operator $1 / \sqrt{2 \hbar}(\hat{X}-$ $\left.i \hat{\Pi}_{X}\right)$. However, how is the other building block included? In other words, where is the black hole entropy in all this? It was suggested in [39] to include another operator representing the internal degrees of freedom (similar to the "secret" operator described in [47]). In the following, we present another way to include the second building block.

Carlip and Teitelboim [41] have shown that the standard (Euclidean) action principle for the gravitational field implies that for spacetimes with black hole topology, the opening angle $\Theta$ at the horizon and the horizon area $A / 8 \pi$ are canonical conjugates. They have argued that the boundary term at the horizon, $-A \delta \Theta$, should supplement the canonical action just as the boundary term at infinity, $M \delta T$, does $\left(T \equiv P_{M}\right.$, the time separation at spatial infinity, is the canonical conjugate to the ADM mass). Classically, $A=16 \pi M^{2}$ and $\Theta=\kappa P_{M}$ (see the last paragraph of this section); however, the above argument motivates us to consider (initially) $M, P_{M}, A / 8 \pi$ and $\Theta$ as if they were four independent parameters. Only at the end, after quantization, do we impose the classical relation. This technique has appeared also in our grand canonical approach [26]. Adding to Eq. (12) the canonical transformation $Y=\sqrt{A / 4 \pi} \cos \Theta$ and $\Pi_{Y}=\sqrt{A / 4 \pi} \sin \Theta$, implies that

$$
\begin{aligned}
16 \pi M^{2} & =4 \pi\left(X^{2}+\Pi_{X}^{2}\right) \\
A & =4 \pi\left(Y^{2}+\Pi_{Y}^{2}\right) .
\end{aligned}
$$

Thus, after quantization, the two "building blocks" $\hat{a} \equiv 1 / \sqrt{2 \hbar}\left(\hat{X}-i \hat{\Pi}_{X}\right)$ and $\hat{b} \equiv$ $1 / \sqrt{2 \hbar}\left(\hat{Y}-i \hat{\Pi}_{Y}\right)$ raise $16 \pi \hat{M}^{2}$ and $\hat{A}$, respectively, by one unit. Since $\hat{A}$ and $\hat{M}$ are considered independent, so are $\hat{X}$ and $\hat{Y}$, and thus $[\hat{a}, \hat{b}]=0$. However, since classically $A$ and $M$ are not independent, one must impose a certain condition relating $\hat{A}$ and $\hat{M}$ (this is analogous to the requirement of zero hyperspin). After imposing this condition $\hat{a}$ and $\hat{b}$ no longer commute, in harmony with the algebraic approach [17]. In this way we have obtained two building blocks: one is associated with the boundary at infinity through $M$ and $P_{M}$, and the other with the boundary at the horizon via $A$ and $\Theta$. Thus, by a comparison with the reduced phase space approach, we have found that there are exactly two building blocks, i.e. $k=2$.

It is interesting to note that the "opening angle" defined classically by Carlip and Teitelboim is related to $M$ and $P_{M}$ by $\Theta=\kappa P_{M}$. This follows from

$$
1=\frac{1}{8 \pi}\{A, \Theta\}_{M, P_{M}} \equiv \frac{1}{8 \pi}\left(\frac{\partial A}{\partial M} \frac{\partial \Theta}{\partial P_{M}}-\frac{\partial A}{\partial P_{M}} \frac{\partial \Theta}{\partial M}\right),
$$

\footnotetext{
${ }^{1}$ In Eq.( 13) $\kappa P_{M}$ is denoted by $\Theta$; here we show that it is actually the "opening angle" defined by Teitelboim and collaborators
} 
where the Poisson brackets are taken with respect to the canonical coordinates $\left(M, P_{M}\right)$. Now, since $A=16 \pi M^{2}$ is independent of $P_{M}$, Eq. (15) implies that $\partial \Theta / \partial P_{M}=8 \pi \partial M / \partial A=$ $\kappa$. Hence, $\Theta=\kappa P_{M}$, and since $\Theta$ has a period $2 \pi$, the Euclidean time $P_{M}$ has a period $2 \pi / \kappa$. In this way we derive the Hawking temperature, which according to finite temperature quantum field theory is equal to $\hbar$ divided by the Euclidean period $2 \pi / \kappa$.

\section{THE HYPERSPIN WITHIN THE ALGEBRAIC APPROACH}

In previous work [17], it was shown that the algebra of the two building blocks (see Eq. (8-10)) leads to $2^{n}$-fold degeneracy of the $n$-th area level. Thus, states with the same area eigenvalue $n a_{0}$ could be represented in a binary form:

$$
\left.\left|x_{1} x_{2} \cdots x_{n}\right\rangle\right\rangle \equiv \hat{x}_{1} \hat{x}_{2} \cdots \hat{x}_{n}|\operatorname{vac}\rangle
$$

where $x_{i}=0$ or 1 and correspondingly $\hat{x}_{i}$ is either $\hat{a}$ or $\hat{b}(i=1,2, ., n)$. As we shall see in the following, not all the states in Eq. (16) correspond to zero hyperspin.

The commutation relations in Eq. (8-10) are invariant under the transformation:

$$
\left(\begin{array}{l}
\hat{a} \\
\hat{b}
\end{array}\right) \rightarrow\left(\begin{array}{ll}
u_{11} & u_{12} \\
u_{21} & u_{22}
\end{array}\right)\left(\begin{array}{l}
\hat{a} \\
\hat{b}
\end{array}\right) \equiv \mathbf{u}\left(\begin{array}{l}
\hat{a} \\
\hat{b}
\end{array}\right)
$$

where $\mathbf{u} \in U(2)$ is a 2-dimensional unitary matrix (here boldface indicates matrices). The group $U(2)$ can be written as a direct product of $U(1)$ and $S U(2)$, i.e. $U(2)=U(1) \times S U(2)$. It follows from Eq. (8) that

$$
\begin{aligned}
& \exp (-i \theta \hat{N}) \hat{a} \exp (i \theta \hat{N})=\exp (-i \theta) \hat{a} \\
& \exp (-i \theta \hat{N}) \hat{b} \exp (i \theta \hat{N})=\exp (-i \theta) \hat{b} .
\end{aligned}
$$

Hence, the generator of the $U(1)$ symmetry is the area operator $\hat{N}$ itself. We shall denote the generators of the $S U(2)$ symmetry by $\overrightarrow{\mathcal{J}}=\left(\hat{\mathcal{J}}_{1}, \hat{\mathcal{J}}_{2}, \hat{\mathcal{J}}_{3}\right)$ and we shall call $\overrightarrow{\mathcal{J}}$ the (total) hyperspin of the black hole. Thus, the hyperspin arise as a result of the symmetry between the two building blocks $\hat{a}$ and $\hat{b}$. Since the hyperspin generates the $S U(2)$ symmetry,

$$
\left[\hat{\mathcal{J}}_{i}, \hat{\mathcal{J}}_{j}\right]=i \varepsilon_{i j k} \hat{\mathcal{J}}_{k} .
$$

The commutation relations between $\hat{\mathcal{J}}_{k}(k=1,2,3)$ and $\hat{a}$ (or $\left.\hat{b}\right)$, can be deduced from the definition of $\overrightarrow{\mathcal{J}}$ as the generators of the symmetry group. That is,

$$
\left(\begin{array}{c}
\exp \left(-i \theta \hat{\mathcal{J}}_{k}\right) \hat{a} \exp \left(i \theta \hat{\mathcal{J}}_{k}\right) \\
\exp \left(-i \theta \hat{\mathcal{J}}_{k}\right) \hat{b} \exp \left(i \theta \hat{\mathcal{J}}_{k}\right)
\end{array}\right)=\exp \left(i \theta \sigma_{k}\right)\left(\begin{array}{c}
\hat{a} \\
\hat{b}
\end{array}\right)
$$

where $\left\{\sigma_{k}\right\}$ are the three Pauli matrices. Now, taking the derivative with respect to $\theta$ in both sides of Eq. (20), and then setting $\theta=0$ we find that:

$$
\begin{array}{ll}
{\left[\hat{\mathcal{J}}_{1}, \hat{a}\right]=\frac{1}{2} \hat{b} \quad,} & {\left[\hat{\mathcal{J}}_{1}, \hat{b}\right]=\frac{1}{2} \hat{a}} \\
{\left[\hat{\mathcal{J}}_{2}, \hat{a}\right]=\frac{i}{2} \hat{b} \quad,} & {\left[\hat{\mathcal{J}}_{2}, \hat{b}\right]=-\frac{i}{2} \hat{a}} \\
{\left[\hat{\mathcal{J}}_{3}, \hat{a}\right]=\frac{1}{2} \hat{a}} & ,
\end{array}
$$


These commutation relations determine the hyperspin uniquely. For example, the third component of the hyper-spin measure the difference between the number of $a$ 's and the number of $b$ 's divided by two; i.e.

$$
\hat{\mathcal{J}}_{3}=\frac{1}{2}\left(\hat{N}_{a}-\hat{N}_{b}\right)
$$

where $\hat{N}_{a} \equiv \hat{N}-\hat{N}_{b}$ and $\hat{N}_{b}$ is defined by

$$
\left.\left.\hat{N}_{b}\left|x_{1} x_{2} \cdots x_{n}\right\rangle\right\rangle=\left(\sum_{i=1}^{n} x_{i}\right)\left|x_{1} x_{2} \cdots x_{n}\right\rangle\right\rangle .
$$

\section{A. The hyperspin representation}

The $2^{n}$ states defined in Eq. (16) span the Hilbert space of all states representing a black hole with an area $a_{0} n$. Here we would like to classify these states according to their hyperspin. Henceforth the states in the hyperspin representation will be denoted by $\left|n, \mathcal{J}, \mathcal{J}_{3}, s\right\rangle$. Eq. (19) implies that $\mathcal{J}$ and $\mathcal{J}_{3}$ must be integers or half integers. Further, according to Eq. (22) the maximum possible value of $\mathcal{J}_{3}$ (and therefore of $\mathcal{J}$ ) is $n / 2$. Thus, $\mathcal{J}=0,1,2, \ldots, n / 2$ (if $n$ is even) or $\mathcal{J}=1 / 2,3 / 2, \ldots, n / 2$ (if $n$ is odd) and $\mathcal{J}_{3}=-\mathcal{J},-\mathcal{J}+1, \ldots, \mathcal{J}$. The quantum number $s=0,1, \ldots, g\left(n, \mathcal{J}, \mathcal{J}_{3}\right)-1$ distinguishes between states with the same area and the same hyperspin; $g\left(n, \mathcal{J}, \mathcal{J}_{3}\right)$ is the number of independent states with the same area level and the same hyperspin (later we shall see that $g\left(n, \mathcal{J}, \mathcal{J}_{3}\right)$ does not actually depend on $\left.\mathcal{J}_{3}\right)$. Thus, the set of states $\left\{\left|n, \mathcal{J}, \mathcal{J}_{3}, s\right\rangle\right\}$ represents an orthonormal basis for the black hole Hilbert space.

We shall mention here that Eq. (22) also implies that for a given $\mathcal{J}$ the minimum $n$ is $2 \mathcal{J}$. Therefore, the area spectrum can be written as

$$
A_{l, \mathcal{J}}=a_{0}(l+2 \mathcal{J})
$$

where $l=0,1,2, \ldots$ However, since the hyperspin of a black hole which was formed by a gravitational collapse is zero (see Sec. [1] and Sec. V) this spectrum coincide with Eq. (2).

The $n=0$ area level (the vacuum state $|v a c\rangle$ ) has zero hyperspin and it is denoted here by $|0,0,0,0\rangle$. The $n=1$ area level has two independent states: $|1,1 / 2,1 / 2,1\rangle \equiv|0\rangle\rangle \equiv \hat{a}|v a c\rangle$ and $|1,1 / 2,-1 / 2,1\rangle \equiv|1\rangle\rangle \equiv \hat{b}|v a c\rangle$.

The $n=2$ area level has 4 independent (orthonormal) states. One state with zero hyperspin and three with $\mathcal{J}=1$ :

$$
\begin{aligned}
|2,1,-1,1\rangle & \left.=\frac{1}{\sqrt{2+w}}|11\rangle\right\rangle \\
|2,1,0,1\rangle & \left.\left.=\frac{1}{\sqrt{4+2 w}}(|01\rangle\rangle+|10\rangle\right\rangle\right) \\
|2,1,1,1\rangle & \left.=\frac{1}{\sqrt{2+w}}|00\rangle\right\rangle \\
|2,0,0,1\rangle & \left.\left.=\frac{1}{\sqrt{2 w}}(|01\rangle\rangle-|10\rangle\right\rangle\right) .
\end{aligned}
$$


Note that $s=1$ in all the states in Eq. (25) since $g\left(n=2, \mathcal{J}, \mathcal{J}_{3}\right)=1$ for all $\mathcal{J}=$ $0,1 \mathcal{J}_{3}=1,0,-1$. However, this is no longer true for $n>2$. For example, using the commutation relations (21), it can be shown that the two independent states $|010\rangle\rangle-|001\rangle\rangle$ and $|100\rangle\rangle-|010\rangle\rangle$ both have $n=3, \mathcal{J}=1 / 2$ and $\mathcal{J}_{3}=1 / 2$. We shall turn now to calculate explicitly the number of independent states with the same $n$ and $\mathcal{J}$.

\section{B. Degeneracy in the hyperspin representation}

First, we shall show that $g\left(n, \mathcal{J}, \mathcal{J}_{3}\right)$ is not a function of $\mathcal{J}_{3}$. In order to prove that, consider the $g\left(n, \mathcal{J}, \mathcal{J}_{3}\right)$ independent states

$$
\left\{\left|n, \mathcal{J}, \mathcal{J}_{3}, s\right\rangle\right\}_{s=0,1, \ldots, g\left(n, \mathcal{J}, \mathcal{J}_{3}\right)-1}
$$

As mentioned above, states with different $s$ are orthogonal. If $\mathcal{J}_{3} \neq \pm \mathcal{J}$ then $\hat{\mathcal{J}}_{ \pm}\left|n, \mathcal{J}, \mathcal{J}_{3}, s\right\rangle \neq 0$ for all $s=0,1, \ldots, g\left(n, \mathcal{J}, \mathcal{J}_{3}\right)-1$, where $\hat{\mathcal{J}}_{ \pm} \equiv \hat{\mathcal{J}}_{1} \pm i \hat{\mathcal{J}}_{2}$. Now, the third component of the hyperspin of all the $g\left(n, \mathcal{J}, \mathcal{J}_{3}\right)$ states

$$
\left\{\hat{\mathcal{J}}_{+}\left|n, \mathcal{J}, \mathcal{J}_{3}, s\right\rangle\right\}_{s=0,1, \ldots, g\left(n, \mathcal{J}, \mathcal{J}_{3}\right)-1}
$$

is $\mathcal{J}_{3}+1$. Furthermore, these states are also orthogonal because

$$
\begin{aligned}
\left\langle n, \mathcal{J}, \mathcal{J}_{3}, s^{\prime}\left|\hat{\mathcal{J}}_{+}^{\dagger} \hat{\mathcal{J}}_{+}\right| n, \mathcal{J}, \mathcal{J}_{3}, s\right\rangle & =\left\langle n, \mathcal{J}, \mathcal{J}_{3}, s^{\prime}\left|\left(\hat{\mathcal{J}}^{2}-\hat{\mathcal{J}}_{3}^{2}-\hat{\mathcal{J}}_{3}\right)\right| n, \mathcal{J}, \mathcal{J}_{3}, s\right\rangle \\
& =\left(\mathcal{J}(\mathcal{J}+1)-\mathcal{J}_{3}^{2}-\mathcal{J}_{3}\right)\left\langle n, \mathcal{J}, \mathcal{J}_{3}, s^{\prime} \mid n, \mathcal{J}, \mathcal{J}_{3}, s\right\rangle=0
\end{aligned}
$$

for $s \neq s^{\prime}$. In the same way it can be shown that the $g\left(n, \mathcal{J}, \mathcal{J}_{3}\right)$ states $\left\{\hat{\mathcal{J}}_{-}\left|n, \mathcal{J}, \mathcal{J}_{3}, s\right\rangle\right\}$ are all orthogonal with $\mathcal{J}_{3}-1$. Hence, $g\left(n, \mathcal{J}, \mathcal{J}_{3}\right)=g\left(n, \mathcal{J}, \mathcal{J}_{3}+1\right)=g\left(n, \mathcal{J}, \mathcal{J}_{3}-1\right) \equiv g(n, \mathcal{J})$. Note that $g(n, \mathcal{J})$ counts the number of states with the same $n, \mathcal{J}$ and $\mathcal{J}_{3}$, even though it does not depend on $\mathcal{J}_{3}$.

According to Eq. (22)

$$
N_{b}=\frac{1}{2} n-\mathcal{J}_{3}
$$

because $N_{a}+N_{b}=n$. Thus, the number of independent states with the same area $n$ and the same $\mathcal{J}_{3}$ is $\left(\begin{array}{c}n \\ N_{b}\end{array}\right)=\left(\begin{array}{c}n \\ n / 2-\mathcal{J}_{3}\end{array}\right)$. For example, the maximum value of $\mathcal{J}_{3}$ is $n / 2$, and in this case $N_{a}=n$ and $N_{b}=0$. Thus, there is only one independent state with area $n$ and with $\mathcal{J}_{3}=n / 2$; that is the state $\left.|00 \ldots 0\rangle\right\rangle$. This implies that $g(n, \mathcal{J}=n / 2)=\left(\begin{array}{l}n \\ 0\end{array}\right)=1$ because $g(n, \mathcal{J}=n / 2) \equiv g\left(n, \mathcal{J}=n / 2, \mathcal{J}_{3}\right)$ is independent of $\mathcal{J}_{3}$ and for $\mathcal{J}=n / 2$ we can set (for convenience) $\mathcal{J}_{3}=n / 2$.

The number of states with $\mathcal{J}_{3}=n / 2-1\left(N_{b}=1\right)$ is equal to $\left(\begin{array}{l}n \\ 1\end{array}\right)$. Now, $g(n, \mathcal{J}=n / 2)$ is the number of states with $\mathcal{J}=n / 2$ and a particular $\mathcal{J}_{3}$; in particular it is also the number states with $\mathcal{J}=n / 2$ and $\mathcal{J}_{3}=n / 2-1$ (remember that $g(n, \mathcal{J}=n / 2) \equiv g\left(n, \mathcal{J}=n / 2, \mathcal{J}_{3}\right)$ for any $\mathcal{J}_{3}$ ). Therefore,

$$
g(n, \mathcal{J}=n / 2-1)=\left(\begin{array}{l}
n \\
1
\end{array}\right)-g(n, \mathcal{J}=n / 2)=\left(\begin{array}{l}
n \\
1
\end{array}\right)-\left(\begin{array}{l}
n \\
0
\end{array}\right)
$$


Similarly, in order to obtain $g(n, \mathcal{J}=n / 2-2)$, we have to subtract from the $\left(\begin{array}{l}n \\ 2\end{array}\right)$ states with the same $\mathcal{J}_{3}=n / 2-2$ the number of states with $\mathcal{J}=n / 2-1$ and $\mathcal{J}=n / 2$. That is,

$$
\begin{aligned}
g(n, \mathcal{J}=n / 2-2) & =\left(\begin{array}{l}
n \\
2
\end{array}\right)-g(n, \mathcal{J}=n / 2-1)-g(n, \mathcal{J}=n / 2) \\
& =\left(\begin{array}{l}
n \\
2
\end{array}\right)-\left[\left(\begin{array}{l}
n \\
1
\end{array}\right)-\left(\begin{array}{l}
n \\
0
\end{array}\right)\right]-\left(\begin{array}{l}
n \\
0
\end{array}\right)=\left(\begin{array}{l}
n \\
2
\end{array}\right)-\left(\begin{array}{l}
n \\
1
\end{array}\right)
\end{aligned}
$$

Continuing in the same way, we find that

$$
g(n, \mathcal{J})=\left(\begin{array}{c}
n \\
n / 2-\mathcal{J}
\end{array}\right)-\left(\begin{array}{c}
n \\
n / 2-(\mathcal{J}+1)
\end{array}\right)=\frac{2 \mathcal{J}+1}{m+\mathcal{J}+1}\left(\begin{array}{c}
2 m \\
m+\mathcal{J}
\end{array}\right)
$$

where $m \equiv n / 2$. Note that by setting the hyperspin to zero Eq. (6) is obtained. In the Appendix we generalize Eq. (30) assuming the algebra is constructed from three building blocks.

The requirement that $\mathcal{J}=0$ implies that the states describing the black hole have a definite "parity". In our algebra, the operator $\hat{p}$ that exchanges the two building blocks is called the parity operator:

$$
\left.\left.\hat{p}\left|x_{1} x_{2} \ldots x_{n}\right\rangle\right\rangle=\left|\bar{x}_{1} \bar{x}_{2} \ldots \bar{x}_{n}\right\rangle\right\rangle,
$$

where $\bar{x}_{i}=1-x_{i}(i=1,2, \ldots, n)$. It can be shown the the parity operator commutes with

$\overrightarrow{\mathcal{J}}^{2}$ but anticommutes with $\hat{\mathcal{J}}_{3}$. Therefore, the only states with definite $\mathcal{J}$ and $\mathcal{J}_{3}$ that have a definite parity are states with zero hyperspin. Also in the framework of the canonical quantization of a Schwarzschild black hole 48 it was shown that the relevant quantum states that exhibit the equally spaced area spectrum have a definite parity. Hence, imposing on the quantum states the requirement of a definite parity is translated in our approach as the requirement that the hyperspin is zero.

\section{DISCUSSION}

Most (and perhaps all) of the observables in physics, such as momentum, angular momentum and energy arise from some symmetry transformations. The hyperspin defined in the previous sections is also derived in the same manner. In the algebra addressed in the previous paper [17], we have used two building blocks $\hat{a}$ and $\hat{b}$. The symmetry between $\hat{a}$ and $\hat{b}$ implies the existence of the hyperspin. In harmony with the heuristic approach it is an observable which represents a global quantum number of the black hole.

In our algebra we have obtained the "maximum" symmetry between the two building blocks; i.e. $U(2)$ symmetry. We cannot obtain a larger group because there are only two building blocks. In the Appendix, we discuss the algebra of three building blocks, which, therefore, has a larger symmetry. It is very natural that in the construction of an algebra describing the inner structure of a black hole some other global quantum numbers will appear. The question is then, how can it live in peace with the "no hair" principles?

The only way to resolve this issue is to set the hyperspin to zero. Since we consider here a neutral non-rotating black hole, the hyperspin cannot represent the angular momentum (or 
charge) of the black hole. Further, the entropy will not be a function just of the horizon area if one considers the hyperspin as the angular momentum. Similar arguments imply that the hyperspin cannot represent quantum numbers like charm or strangeness of ordinary matter; otherwise it must be very small to be compatible with the classical limit. Therefore, it is reasonable to assume that the hyperspin represents a quantum number which does not exist in ordinary matter. The conservation of the hyperspin then implies that a black hole which was formed by a gravitational collapse of ordinary matter must have zero hyperspin.

Hawking showed very convincingly that at the semi-classical level, where the gravitational field is treated classically but the matter fields exterior to the black hole are quantized, black holes radiate all kinds of particles with spectrum of a black body. If these particles do not possess hyperspin, but the black hole does, the Hawking radiation must cease at the point when the horizon area becomes equal to the hyperspin (see Eq. (24)). Thus, eternal black holes which do not radiate can have $\mathcal{J} \gg 1$. Of course, this hyperspin can not be measured by an observer outside the black hole.

We conclude that the hyperspin cannot represents a quantum number of ordinary matter. Instead, it might represent a global feature of the $D_{0}$-branes in $M$-theory, the Wilson lines in the Ashtekar approach, or a quantum number attached to the patches described in the heuristic approach.

\section{ACKNOWLEDGMENTS}

I would like to thank J. Bekenstein for his guidance and support. This research was partly supported by grant No. 129/00-1 of the Israel Science Foundation and by a Clore Foundation fellowship. The author is also grateful for the Killam Trust for its financial support.

\section{APPENDIX A: THE ALGEBRA OF THREE BUILDING BLOCKS}

The algebra of two building blocks (8)-(10) can be generalized to that with three building blocks. Adding another building block, say $\hat{c}$, one must assume the following commutation relations:

$$
\begin{aligned}
& \text { (1) }[\hat{A}, \hat{c}]=a_{0} \hat{c} \\
& \text { (2) }\left[\hat{c}^{\dagger}, \hat{c}\right]=1+w \hat{N} \\
& \text { (3) }\left[\hat{c}^{\dagger}, \hat{a}\right]=\left[\hat{c}^{\dagger}, \hat{b}\right]=0
\end{aligned}
$$

These commutation relations follows from the same arguments that led to Eq. (8-10) (see [17]), and together with Eq. (8-10) constitute the totality algebra of three building blocks. The algebra is invariant under the transformation:

$$
\left(\begin{array}{c}
\hat{a} \\
\hat{b} \\
\hat{c}
\end{array}\right) \rightarrow\left(\begin{array}{lll}
v_{11} & v_{12} & v_{13} \\
v_{21} & v_{22} & v_{23} \\
v_{31} & v_{32} & v_{33}
\end{array}\right)\left(\begin{array}{c}
\hat{a} \\
\hat{b} \\
\hat{c}
\end{array}\right) \equiv \mathbf{v}\left(\begin{array}{c}
\hat{a} \\
\hat{b} \\
\hat{c}
\end{array}\right),
$$

where $\mathbf{v} \in U(3) \equiv U(1) \times S U(3)$ is a 3 -dimensional unitary matrix. The generator of the $U(1)$ symmetry is the area operator $\hat{N}$ itself. 
The group $S U(3)$ has 8 generators which are denoted here by $\hat{\mathcal{G}}_{k}(k=1,2, \ldots, 8)$ (a basis of the $S U(3)$-Lie algebra generalizing the Pauli matrices is given by the 8 Gell-Mann matrices [49]). The commutation relations between $\hat{\mathcal{G}}_{k}(k=1,2, \ldots, 8)$ and $\hat{a}($ or $\hat{b})$, can be deduced from

$$
\left(\begin{array}{l}
\exp \left(-i \theta \hat{\mathcal{G}}_{k}\right) \hat{a} \exp \left(i \theta \hat{\mathcal{G}}_{k}\right) \\
\exp \left(-i \theta \hat{\mathcal{G}}_{k}\right) \hat{b} \exp \left(i \theta \hat{\mathcal{G}}_{k}\right) \\
\exp \left(-i \theta \hat{\mathcal{G}}_{k}\right) \hat{c} \exp \left(i \theta \hat{\mathcal{G}}_{k}\right)
\end{array}\right)=\exp \left(i \theta \lambda_{k}\right)\left(\begin{array}{c}
\hat{a} \\
\hat{b} \\
\hat{c}
\end{array}\right),
$$

where $2\left\{\lambda_{k}\right\}$ are the eight Gell-Mann matrices that generalizing the Pauli matrices 44. The first three generators $\mathcal{G}_{1}, \mathcal{G}_{2}$ and $\mathcal{G}_{3}$ satisfy the same commutation relations given in Eq. (21) if one replace $\mathcal{J}_{k}$ by $\mathcal{G}_{k}$ for $k=1,2,3$. Therefore, we shall identify the first three generators with the hyperspin, i.e. $\mathcal{G}_{k} \equiv \mathcal{J}_{k}$ for $k=1,2,3$. In addition, from Eq. (A3) it follows that the third building block $\hat{c}$ commutes with the hyperspin $([\hat{c}, \overrightarrow{\mathcal{J}}]=0)$.

Further, Eq. (A3) implies that the eight generator $\mathcal{G}_{8} \equiv \hat{Y}$ (similar to the hypercharge in the standard model) commutes with the hyperspin and satisfies the commutation relations:

$$
[\hat{Y}, \hat{a}]=\frac{1}{2 \sqrt{3}} \hat{a}, \quad[\hat{Y}, \hat{b}]=\frac{1}{2 \sqrt{3}} \hat{b} \quad \text { and } \quad[\hat{Y}, \hat{c}]=-\frac{1}{\sqrt{3}} \hat{c} .
$$

That is, $\hat{Y}=\frac{1}{2 \sqrt{3}}\left(\hat{N}_{a}+\hat{N}_{b}-2 \hat{N}_{c}\right)$.

We can define $3^{n}$ states with the same area just as in Eq. (25), except that now $x_{i}$ can have the values $-1,0$ or 1 and correspondingly $\hat{x}_{i}$ is either $\hat{a}, b$ or $\hat{c}$. It can be shown that all the $3^{n}$ states are independent (for two building blocks see the proof in [17]). Now, since $\hat{N}, \overrightarrow{\mathcal{J}}^{2}, \hat{\mathcal{J}}_{3}$ and $\hat{Y}$ all commute with each other, we can write the $3^{n}$ black hole states in the form $\left|n, \mathcal{J}, \mathcal{J}_{3}, Y, s\right\rangle$, where $\mathcal{J}=0,1,2, \ldots, n / 2$ (if $n$ is even) or $\mathcal{J}=1 / 2,3 / 2, \ldots, n / 2$ (if $n$ is odd), $\mathcal{J}_{3}=-\mathcal{J},-\mathcal{J}+1, \ldots, \mathcal{J}, 2 \sqrt{3} Y=-n,-n+3,-n+6, \ldots, 2 n$ and $s=$ $0,1, \ldots, g(n, \mathcal{J}, Y)-1$ where $g(n, \mathcal{J}, Y)$ is the number of states with the same area, hyperspin $\mathcal{J}, \mathcal{J}_{3}$ and "hypercharge" $Y$.

By using the same technique that led to Eq. (30), one can show that

$$
g(n, \mathcal{J}, Y)=\frac{2 \mathcal{J}+1}{\eta+y+\mathcal{J}+1}\left(\begin{array}{c}
3 \eta \\
\eta+y-\mathcal{J}
\end{array}\right)\left(\begin{array}{c}
2 \eta-y+\mathcal{J} \\
\eta-2 y
\end{array}\right)
$$

where $\eta \equiv n / 3$ and $y \equiv Y / \sqrt{3}$. Now, since the hyperspin $\mathcal{J}$ and also $Y$ are zero (see Sec. Ø), the degeneracy of the $n$th area level is given by:

$$
g(n)=\frac{1}{1+\eta}\left(\begin{array}{c}
3 \eta \\
\eta
\end{array}\right)\left(\begin{array}{c}
2 \eta \\
\eta
\end{array}\right)
$$

where we set $\mathcal{J}=Y=0$ in Eq. (A5). Using Stirling's formula, in the limit $n \rightarrow \infty$

$$
g(n) \approx \frac{3^{n}}{n^{2}}
$$

Hence, in the case of three building blocks the leading correction term to the BekensteinHawking entropy is $-2 \ln n$. 


\section{REFERENCES}

[1] R. M. Wald, Living Reviews in Relativity, gr-qc/9912119.

[2] J. D. Bekenstein Phys. Rev. D, 7, 2333 (1973).

[3] M. B. Green, J. H. Schwarz and E. Witten, Superstring theory (Cambridge University Press, Cambridge 1987); J. Polchinski, String Theory (Cambridge University Press, Cambridge 1998).

[4] A. Ashtekar and K. Krasnov, in Black Holes, Gravitational Radiation and the Universe, B. R. Iyer and B. Bhawal eds. (Kluwer, Dordrecht 1998), 149-170; A. Ashtekar, C. Rovelli and L. Smolin, Phys. Rev. Letters 69, 237 (1992).

[5] S. Weinberg, in General Relativity: An Einstein Centenary Survey, S. W. Hawking and W. Israel, eds. (Cambridge University Press, Cambridge 1979); K. Kuchař, gr-qc/9304012.

[6] T. Jacobson, gr-qc/9404039; V. P. Frolov, D. V. Fursaev and A. I. Zelnikov, Nucl.Phys. B486, 339 (1997); V. P. Frolov and D. V. Fursaev, Phys.Rev. D56, 2212 (1997); V. P. Frolov and D. V. Fursaev, hep-th/9705207.

[7] S. Carlip, Phys.Rev.Lett. 88, 241301 (2002); S. N. Solodukhin, Phys.Lett. B454, 213 (1999).

[8] G. Gour and A. E. Mayo, Phys. Rev. D63, 064005 (2001).

[9] R. K. Kaul and P. Majumdar, Phys. Rev. Lett. 84, 5255 (2000).

[10] R. K. Kaul and P. Majumdar, Phys. Lett. B439, 267 (1998).

[11] S. Das, K. Kaul and P. Majumdar, Phys. Rev. D 63, 044019 (2001), hep-th/0006211; P. Majumdar, Pramana 55, 511 (2000); hep-th/0011284; hep-th/0110198.

[12] T. R. Govindarajan, R. K. Kaul and V. Suneeta, Class.Quant.Grav. 18, 2877 (2001).

[13] S. Carlip, Class. Quant. Grav. 17, 4175 (2000).

[14] S. Das, P. Majumdar and R. K. Bhaduri, Class. Quant. Grav. 19, 2355 (2002); S. Das, hep-th/0207072.

[15] K. S. Gupta, hep-th/0204137 (see also K. S. Gupta and S. Sen, Phys. Lett. 526, 121 $(2002))$.

[16] J. D. Bekenstein, (2002) in 2001: A Spacetime Odyssey, M. Duff and J. T. Liu, eds. (World Scientific Publishing, Singapore 2002); hep-th/0107045.

[17] J. D. Bekenstein and G. Gour, Phys. Rev. D 66, 024005 (2002); gr-qc/0202034.

[18] S. N. Solodukhin, Phys. Rev. D 51, 609 (1995); Phys. Rev. D 51, 618 (1995); Phys. Rev. D57, 2410 (1998); R. B. Mann and S. N. Solodukhin, Nucl. Phys. B 523, 293 (1998).

[19] D. Fursaev, Phys. Rev. D51, 5352 (1995).

[20] J. Jing, M-L Yan, Phys. Rev. D63, 24003(2001).

[21] D. Birmingham, S. Sen, Phys. Rev. D63, 47501 (2001); D. Birmingham, I. Sachs, S. Sen, Int. J. Mod. Phys. D10, 833 (2001).

[22] C. O. Lousto, Phys. Lett. B 352, 228 (1995).

[23] S. Major and K. Setter, Class. Quant. Grav. 18, 5125 (2001); Class .Quant. Grav. 18, 5293 (2001).

[24] H. A. Kastrup, Phys. Lett. B 413, 267 (1997).

[25] J. Mäkelä and P. Repo, How to interpret black hole entropy, gr-qc/9812075.

[26] G. Gour, Phys. Rev. D 61, 021501(R) (1999).

[27] O. Obregon, M. Sabido and V. I. Tkach, Gen. Rel. Grav. 33, 913 (2001).

[28] J. D. Bekenstein, Lett.Nuovo Cimento 11, 467 (1974). 
[29] J. D. Bekenstein, in Black Holes, Gravitational Radiation and the Universe, B. R. Iyer and B. Bhawal eds. (Kluwer, Dordrecht 1998), pp. 87-101.

[30] A. E. Mayo, Phys. Rev. D58 104007 (1998).

[31] J. D. Bekenstein, in IX Brazilian School of Cosmology and Gravitation, M. Novello, ed. (Atlantiscience, Paris 1999); gr-qc/9808028.

[32] A. Krause, hep-th/0205310.

[33] V. F. Mukhanov, Pis'ma Zh. Eksp. Teor. Fiz. 44, 50 (1986) [JETP Lett. 44, 63 (1986)].

[34] J. D. Bekenstein and V. F. Mukhanov, Phys. Lett. B 360, 7 (1995).

[35] U. H. Danielsson and M. Schiffer, Phys. Rev. D48, 4779 (1993).

[36] J. A. Wheeler, A Journey into Gravitation and Spacetime (Freeman, New York, 1990).

[37] R. D. Sorkin, in Black Holes and Relativistic Stars, R. M. Wald, ed. (University of Chicago Press, Chicago, 1998).

[38] H. A. Kastrup, Ann. d. Physik. 9, 503 (2000).

[39] S. Das, P. Ramadevi and U. A. Yajnik, Mod. Phys. Lett. A17, 993 (2002).

[40] A. Barvinsky, S. Das and G. Kunstatter, Class. Quant. Grav. 18, 4845 (2001); Phys. Lett. B 517, 415 (2001).

[41] S. Carlip and C. Teitelboim, Class. Quant. Grav. 12, 1699 (1995).

[42] R. Gambini and J. Pullin, Loops, Knots, Gauge Theories and Quantum Gravity, (Cambridge University Press, Cambridge 1996).

[43] T. Banks et al, JHEP 9801, 008 (1998); H. Liu and A. A. Tseytlin, JHEP 9801, 010 (1998).

[44] D. Louis-Martinez and G. Kunstatter, Phys. Rev. D 52, 3494 (1995); A. J. M. Medved and G. Kunstatter, Phys. Rev. D 59, 104005 (1999).

[45] K. Kuchař, Phys. Rev. D 50, 3961 (1994).

[46] S. W. Hawking, Euclidean Quantum Gravity in Recent Developments in Gravitation, Cargese Lectures, eds. M. Levy and S. Deser, (Plenum 1978); The Path Integral Approach to Quantum Gravity in General Relativity: An Einstein Centenary Survey eds. Hawking, S. W. and Israel, W. (Cambridge University Press, Cambridge, England 1979).

[47] G. Gour, Phys. Rev. D 61, 124007 (2000).

[48] C. Vaz, Phys. Rev. D 61, 064017 (2000).

[49] See for example C. Itzykson and J. B. Zuber, Quantum Field Theory, p.516 (McGRAWHILL, Singapore, 1980). 